\title{
The 'I' of the author and its persuasive function (as exemplified by the complex sentence with homogeneously collateral sub clauses)
}

\author{
Olga Nikolenko ${ }^{1}$, Olga Zakharchuk ${ }^{1}$, Larisa Babakova ${ }^{1}$, and Boris Morenko ${ }^{1, *}$ \\ ${ }^{1}$ Don State Technical University, Department of Russian as a Foreign Language, 344002, Rostov-on-Don, Russia
}

\begin{abstract}
What makes this study topical: the urgency of the problem under consideration is due to the existing need for structural and semantic analysis of complex sentences (CS) with homogeneously collateral subordination of clauses, in different functional styles of speech and language. Our study is directed towards revealing the ability of syntaxemes with homogeneously collateral subordination to render hidden meanings of the author's 'I' and to thereby affect the reader/hearer. The cornerstone research method in this study is direct observation of language phenomena with generous borrowings from transformational analysis; it allows us to assert that the multi-component sentences under scrutiny here possess powerful expressive potential and can better than any other render additional information, thereby giving a strongly suggestive focus to an utterance or statement. In this paper, for the first time in the history of linguistics, we reveal how CS with homogeneously collateral subordination of sub clauses work in all functional styles. We also define cognitive boundaries within which takes place the choice between such multi-component structures in the process of language activity, with concern for how the 'I' of the author affects the addressee.
\end{abstract}

\section{Introduction}

The complex topography of relations between language and reality, in terms of studying the language image of the world, was what spurred a shift in research interest from the functional aspect to the perceptive/cognitive one.

In recent decades, the notion of a scientific paradigm has been contrapuntally introduced by psycholinguistics, suggestive linguistics, language pragmatics, phonology, mass communication theory, conflict resolution studies. The subject matter of all these sciences is language persuasion as a way of effective communication [1] consisting of "...distinctly set communicative goals and adequate language means" [2], and also the effect of speech uttered with the aim of inciting one person consciously adopt the viewpoint of the other [3].

This can be accounted for by a desire we find in the society to look at language not as solely an instrument of cognition and knowledge and the means of communicating data on this world, but also as a tool empowering us to persuade people, to exert influence upon the linguistic constiousness of a group or an individual, his/her behaviour, thinking and various intellectual activities.

In the modern science of language, persuasion is divided into non-verbal (gestures, mimics, body postures, etc.) and verbal one (speech, discourse, human thinking rendered by language means). Verbal impact is effected by the means of language at all of its levels, and complex sentences with homogeneously collateral sub clauses are of no small importance in this respect. These sintaxemes are more versatile and reach in their semantic and stylistic characteristics because they are 'designed' to be able to express and render very complex grammatical and semantic relations. They are the components of expressive syntax structures, because having the ability to shape speech tactics of the speaker and accommodating a very big expressive charge they can produce a stronger effect on the addressee of an utterance and render, firstly, the inner world of a person, his/her emotions, and secondly, the outer world, i.e. the reality around us in a given situation [4].

Speech tactics are largely dependent on emotivity as a semantic parameter - the one that is, basically, a pragmatically oriented piece of information on what the subject of speech feels about the thought he/she is expressing or the object he/she designates. Linguistic emotivity is a very complex semantic category that renders the emotional impact the addresser has on the addressee through the cognitive content of complicated syntactic structures. In these terms, emotivity is more or less similar to expressivity and includes cognitive/modal/semantic components associated with the sensual, subjective interpretation of the 'status quo'. When we refer to syntactic emotivity we may mean: 1) imagery (knowledge), 2) inner form (opinion), 3) motivation (expressive representation), 4) expressiveness (emotive experience) [5].

In some experiments [6] our colleagues provided a definition of how intentionality/non-intentionality of an

\footnotetext{
*Corresponding author: bmorenko@mail.ru
} 
utterance is related to the cognitive choice of optional syntactic constructions. In terms of the 'language thought - speech' relation, these studies offered some evidence that the pragmatic nature of syntactic units affects intentionality of a sentence and also allows to shift emphases across the utterance, subject to the functional conditions under which it was generated.

In this respect, a study of complex sentences with homogeneously collateral sub clauses in all language styles is what expands this area of research, since it is capable of demonstrating that semantic/syntactic relations do place their mark on intentionality of an utterance subject to which functional style they express themselves in. Besides, cognitive boundaries of the choice of such multi-component structures are determined first in the flow of speech with due consideration of how the 'I' of the author affects the addressee, which appears to be an interesting object for linguistic analysis as it opens up possibilities to observe language phenomena not studied before.

\section{Materials and methods}

As the key general linguistic methodological conceptions, we accept the principles of how the language system and discourse interact. The specific linguistic methodology of our study draws heavily on research in the area of syntax and of the pragmatic characteristics of large syntactic units, such as books and papers by I.A. Vassilenko, G.F. Gavrilov, V.V. Kazmin, G.F. Kalashnikov, V.A. Shitov, et al. The study was carried out as a direct observation of language phenomena with application of a theoretical method (synthesis, instantiation, generalization), as well as the techniques of transformational analysis, transformation and substitution.

The study was done in two steps:

firstly, we analyzed existing methodological approaches to the task of describing complex sentences with homogeneously collateral sub clauses, in order to reveal and identify the cognitive principia discernible in how these syntaxemes vary in the process of speech activity;

at the second step we tried to reveal the specified properties of the multi-component structures under investigation - the ones that make them capable of working in all styles and genres of the language because speech style is determined and specified by the choice of the speaker. We pursued the goal of substantiating the claim that a certain effect is produced by the 'I' of the author in complex sentences with homogeneously collateral sub clauses, and by the stylistic functions of individual optional forms of such complex sentences when they are projected onto the situation of linguistic and extra linguistic personal fulfilment.

\section{Results}

In a scientific text, a series of collateral homogeneous clauses (mainly those of cause, result, condition) do render the train of thought, the continuity, coherence, and unfolding of the speech flow, or the level of detail in the narration: Thus, a system has a single solution if the system's determinant is non-zero and the system is nontrivial [7].

Complex sentences with collateral sub clauses 'squeeze' a large scientific text and line up thoughts and ideas in a logical sequence. The persuasion effect in such structures is produced through the lexically expressed 'I' of the author (parenthetic words, expletives, phrases like "it is known that" or "it is possible to" as correlative words in the main clause) and is more commonly used in natural science texts with an experimental focus: "In science, it is well known that all the effects associated with the emission of large quantities of exchange quanta, are negligibly small, and that simplification may only be fruitful in a theory with a weak constant binding [8].

Many a linguist working in the area of stylistics and studying the scientific style and its properties (S.G. Ilyin, A.N. Surovtsev, V.S. Khrakovsky, et al) do single out a particular group of prepositional-nominal combinations with the preposition 'pri' (Rus. for 'at'). Apartness of such structures may be accounted for by the fact that their respective meanings are undifferentiated, which hinders clear delineation between conditional, temporal and causal relations that can be combined within one syntactic unit. Thanks to their ability to get into synonymic relations, complex sentences with homogeneously collateral sub clauses can overcome language homonymy, specify and thus mark in a very personal way the meaning of a statement: At rises of temperature and heightening of friction, boundary level deformations increase significantly.

Compare: If we raise the temperature and heighten friction, deformations of the boundary level increase (a vividly 'conditional' meaning).

When the temperature rises and friction heightens, deformations of the boundary level increase significantly (the conditional sub clauses are gaining certain 'temporal' overtones) [9].

Using this analysis of syntactic structures in a scientific text, we can make a conclusion that it is multicomponent phrases with collateral sub clauses that can, to a higher degree than other ones, step up the dynamics of narration, emphasize the gist of the text, focus the addressee on the specific component that is necessary for the proper understanding of the text, lead the reader beyond the boundaries of what is directly observable and create for him/her new vast areas of scientific thought. "And such categories as emotionality, expressiveness and evaluativity, being the product of a person's cognition of the world around him/her ... closely interact with bookish words, together making up the expanded additional implicit meanings that amplify the general contents of the phrase..." [10].

Despite the fact that the marked features of the official style are objectivity, brevity of narration, lack of emotion and focus on statement of the fact, such texts also include (although to a much lesser degree) complex sentences with homogeneously collateral sub clauses marked by the 'I' of the author. We have grounds to believe that it can be linked to the fact that this style caters for socio-cultural relations (although it is more 
about business than anything else), and in this realm 'the boss' occupies the central place, while his verbal characteristics do have impact upon psychological atmosphere in the team and on its successes in achieving its goals and tasks. Formatting a thought verbally, the addresser, one way or another, imbues it with his/her subjectivity: language is a universal means of communication; each thought and idea is individualized while respective speech individualities of the participants of an official discourse do have a certain reciprocal effect upon each other: "In order to provide for a more objective assessment of aviation safety situation on the Aeroflot Airlines, and to create conditions for swift responding to actual or potential threats of unlawful interference with the company's operations, Aeroflot representatives suggested introducing amendments to aviation safety plans and procedures" (from a TV report). Surely, complex sentences with collateral sub clauses in the official style may strengthen the personal aspect of speech, overcome its impersonal tendency, fill it with something concrete. In this connection, what one may talk of is that the 'I' of the author and its subjective intentions can be regarded as a certain definite marker of an utterance's expressiveness.

"Journalism is what defines the boundaries of group thinking regulating how and in which direction it is being shaped, reflecting its cognitive, axiological and cultural references and priorities" [11], and this is why it - as a body of stylistic means aimed at implanting special voluntative attitudes in masses of people and in each individual - quickly 'comprehended' and 'took home' even the medical claims that human speech contributes to forming powerful solitary waves that affect practically all living organisms [12]. It is because of this that the majority of modern journalistic texts have as their chief goal not objective communication of information but premeditated, deliberate persuasion: It took me several hours to explain to her that religion and faith are two different notions.

The persuasive side of journalistic style is most vividly represented by complex sentences with homogeneously collateral sub clauses, in the arsenal of which we find all the means necessary to produce a premeditated impact upon the addressee and elicited a response reaction (the one that has been projected by the addresser pre-emptively): Instead of this, they are fully content with what various doctrines are imposing on them, of which they understand none and which do no good to them (from newspaper reports).

The mechanism of speech persuasion in such syntactic structures works along the same scenario: 1) presentation of the information content and 2) disguising what is to be implanted in the recipient's consciousness. At that, it often happens that it is precisely sub clauses that have a significant persuasive potential, whereas the main part of the phrase simply establishes a fact. And the choice of linguistic material is determined by the persuasive 'outcomes' a speaker is striving for, although the general idea is still clearly seen: as a result, a holistic, coherent picture has to be formed in the recipient's mind.

In an attempt to improve their lexical-semantic arsenal needed for a successful communication, many public speakers draw on the cultural heritage and try to project its lessons on modern reality. In this connection, in the science of language there emerged and became clearly felt a new functional style (ecclesiastical and religious) that will cater for the spiritual needs of the society. The ecclesiastical/religious style includes elements of implicit realization of the abstractiveness of this notion as a general humanitarian category. Entering the cross-disciplinary space of both stylistics and syntax, the ecclesiastical style becomes the conceptual and terminological tool that allows us to consider it along with other functional styles and to analyze it as a distinct lexical and semantic unity vested with special modal (substantive and conceptual) and syntactic 'powers' [13]. Being marked by some features of institutional communication, it also demonstrates the properties of interpersonal communication that, unfolding in texts, reflect the inner world of authors and are aimed at shaping one's religious worldview and 'adjusting' the recipients' consciousness: I know that the universe is governed by laws one of which reads: every seed yields its fruit [14].

Comparative analysis of ecclesiastical and religious texts, with the aim of revealing regularities in the use of complex sentences with homogeneously collateral sub clauses, gives us grounds to conclude that many biblical narrations are built as an extended metaphor that, on the one hand, allows one to communicate the contents of divine writings in an accessible form, and, on the other hand, produce a multifaceted effect on the addressee, i.e. to demonstrate how the moral component is being formed - the one oriented, among other things, towards the improvement of the speaker's speech behaviour [15]. The Heavenly Kingdom is like a mustard seed that one has taken and planted on his field, the seed which..., when grown, is higher than all the grasses and becomes a tree whereto fowls of the air fly and hide among its branches (The Gospels, Books of the Old and New Covenant).

"Contraposition between body and soul as something 'high' and 'low' is an invariable of the Christian culture in general" [16]; therefore on the pages of the Bible we see the earthly life unfolding in a struggle against seductions, sins, human fallibility as opposed to the Heavenly life that is a prize for victory over vice in all its manifestations. Hence the motivated use of the means of expressive syntax that chooses structures with adversatives: Thus, I shall compare everyone who listens to these My words and fulfils them to a wise and prudent man who has build his house on stone. And it started raining, and rivers overflowed, and the winds rose and rushed onto that house, but it did not fall because it was based on stone. But each and every one that listens to My words but does not stand by them shall be compared to someone thoughtless and imprudent that has built his house on sand, and the building fell down and its collapse was a great one (The Gospels, Books of the Old and New Covenant).

"Expressive structures are what enriches the system of syntax" [17]; the aptness of syntaxemes with homogeneously collateral sub clauses to render hidden meanings, their vividness and intensity, their imagery is 
what helps religious texts to cloak the key cultural values in a perceptible form, to reflect the emotiveness of the 'I' of the author, and to display unlimited powers of affecting and persuading the reader.

The style of everyday language also bears the stamp of the 'I' of the author since it is invested with a powerful charge of expressiveness and emotionality, which is achieved first and foremost through lexical and intonational means. There is more of a dialog to it, with a tendency towards compaction and the use of laconic, one-word utterances, so multi-components syntactic structures are very few in there. The message of homogeneously collateral sub clauses in this style is weakened to some degree: they sort of merge with the main clause, which leads to structural reduction: Live as you wish and as you can.

Such assimilation demands intonational actualization on the level of spoken language and - on the level of written language - no commas before sub clauses.

Syntactic structures with collateral sub clauses, in the majority of all cases, appear in the spoken language when it comes to descriptions and specification of properties (characteristics) of an object (in a broader sense of nomination) through attributive sub clauses: Today we spoke of the woman, the one - if you remember that - who always wears hats, .... and who was so strange in manner at Andrey's.

Functional styles are not a closed system as they are constantly in the state of active cross-play. The best evidence of this is the fact that we have the belles lettres that, performing both the social and aesthetic functions, 'uses' lexicogrammatical instruments from all the styles. A text of fiction is a "stable and complete system built on the basis of dynamic cross-play of various linguistic elements" [18] and conductive to "... creation of implied sense". [19]; it serves both the social and aesthetic function.

"The relation 'literary work - reader' is a variable subject to many a factor: from individual to social ones". [20]: poets and writers striving for powerful imagery tend to clothe their thoughts in a suitable language form often using unconventional lexemes and syntaxemes:

Wake up and move your shoulder right away, chains,

While you have time and the's no padlock on your

While, at a public sale, by guileful deathman,

You haven't been brought to hammer. Yet. [21].

Complex sentences with homogeneously collateral sub clauses that are more capacious structurally and substantially, in the language of fiction do reflect the multifaceted relations that bind reality together into a unity (which is dictated by the very nature of such syntaxemes):

And I wish them to be all close friends

Your son and my son for the rest of their life,

In weal and in woe together,

Having all shared and shared alike,

So as we can take them to the mountains,

With no shame and disgrace for both of us. [21].

As can be seen in the latter case, persuasiveness is built on multidimensionality of real world phenomena that are linked in the text by punctuation marks, and in speech - by a special type of intonation and by expressiveness that can appear in a communicative act as the means of communicating the speaker's subjective attitude towards the contents of the message or the addressee.

Surely, a much higher frequency of complex sentences with collateral sub clauses is observed in poetry, particularly in poems by $\mathrm{M}$. Lermontov, V. Tsvetaeva, S. Yesenin. In modern poetry we can find these in the works of several poets from Rostov. Linguistic analysis of the works of these outstanding writers, with a focus on the quantitative component of the predicative part within one micro-text expressed by a sentence with collateral clauses, and the functioning in it of co-ordinating and subordinating conjunctions (connective words) was what gave grounds to linguists to conclude that when a subordinating connective appears before each consecutive sub clause, all the elements merge into a coherent whole, that is, sub clauses line up into a uniform syntactic chain, "thanks to which what the text gains is melodiousness and harmony" [22]:

To wish you a sound Caucasian health,

A family of calm and warmth,

To wish you that your star, with love,

Its bread of good should bake for you,

So as your heart should ne'er feel dull or ached,

So as your soul is never cold from chilly winds,

So as your blood should love you as the Sun

That gives us dawns and mornings at the break of day [21].

That such utterances are so easily readable and logically coherent is attained due to the ability of connectives to cumulatively vary the same theme and of the metrical system to provide support to enumeration sequences.

Repetition of subordinating conjunctive elements requires, at the level of spoken language, accentuation and a specific 'suspense'-intonation; in written language it is the intensity originally inherent it the nature of poetry. All this is what allows poets to mark the described reality in peculiarly individual way, to convey its most subtle nuances and engage the reader in this range of thoughts and emotions.

A certain role in the process of creating semantic nuances is given to the synonymy of subordinating connectives: it is what helps both avoid repetitions and form an utterance with an expanded sense, the one that is capable of opening up new and deep shades of meaning "between the lines":

Land of the Urals and its heady perfume,

Where one's inebriated with the air,

And the wood sprite is in the boughs of pines

Telling you good fortunes.

It is where the fresh air fills your chest

And stirs the blood with each and every breath,

Where the dark sky is the face for the eternal compass, the changeless Milky Way [21].

In poetry, on the basis of complex sentences with homogeneously collateral sub clauses, a periodic sentence can be formed - a stylistic device possessing a prominent rhythmical pattern and formal beauty. Its 
succinctness and fullness in rendering the message is what allows a periodic sentence to produce an emotional effect upon the reader, and the utterance thus reaches its emotive completeness [23].

D'you know the wilderness burned by the glaring rays of sun,

With groves and meadows lying withered in their bloom,

Where guile and carelessness pay tribute to malice,

Where hearts of local men are torn by passions,

And where, occasionally, are born

Heads that are cold and firm like stones? [24].

Synonymy of the pronominal adverb 'where' with the connective 'which' is what imbues homogeneous attributive sub clauses with some spatial meaning thus expressively accentuating the 'I' of the author.

\section{Discussions}

The complex sentence with homogeneously collateral sub clauses has been in the focus of linguistic research already since the $1950 \mathrm{~s}$. The linguist who laid the foundations for analysis of such syntactic structures was V.A. Shitov. He singled out three types of complex sentences with collateral sub clauses "on the basis of how conjunctions or conjunctive words are being used, the ones that connect homogeneously collateral sub clauses to the main clause" [25]. What Shitov studies is the problem of conjunction-based connectives' variability and their form; at the same time he does not consider the meanings of conjunction-based connectives and conditions for their necessity.

Regularities in how individual types of complex sentences with homogeneously collateral sub clauses are structured as a special sub-system within the general system of the complex sentence are outlined by G.P. Ukhanov. He was one of the first colleagues who did a quantitative analysis of the use of these syntaxemes in different functional styles.

The problem of variability and synonymy of structures with homogeneously collateral sub clauses was partly considered in the books by G.F.Gavrilova [26] and G.F. Kalashnikova [27]. G.F.Gavrilova carried out system analysis of complex sentences with homogeneously collateral sub clauses in terms of the active (revealing the properties and functions of the mechanism that generates complex sentences and the rules of how the meanings of different syntactic contexts combine) and the passive (revealing the components of a sentence and specifying their functions) syntax [28].

These works were the reference points for our study because they allow us to describe the structures under investigation in terms of how the syntactic meaning is related to its verbal form, i.e. how language form corresponds to the sense rendered by it, and of how relations between the meaning and its syntactic embodiment are inter-determined, the intricate relation between the semantic and structural sides of a linguistic model.

Linguists specializing on syntax do regularly consider a range of problems that, one way or other, are related to the domains of the semantics and complex sentences' structure. The question, however, of how complex sentences with homogeneously collateral sub clauses work in different language styles with respect to the structure of personally marked syntaxemes in particular speech situations, has not been properly studied. In part, this can be accounted for by the fact that structures with homogeneously collateral sub clauses have not yet become the subject of monographs but only individual journal papers.

On the other hand, the four-aspect approach suggested by G.F. Kalashnikova to describing complex syntactic structures, namely: logical, structural, semantic and communicative - paves the way for further studies on structures with homogeneously collateral sub clauses, from the perspective of the actual interplay of linguistic and extra-linguistic factors [27]. More than that: in connection with the above, there arises in syntax studies a need for relying on data derived from beyond the linguistics, first and foremost from the realm of psychology.

Suggestive linguistics that has been developed actively in the recent years entails that the focus of research interest is to be redirected towards the syntactic conditions necessary for action processuality per se and for expressing the 'I' of the author through the specified multi-component syntactic structures, with the aim of producing an effect upon the hearer. Consequently, it cannot be ruled out that the suggestive factor may become a research priority not only for cognitive linguistics but also for syntactic studies.

Besides, it happens every so often that studies of various system relations and links in syntax are based among other things on quantitative methods, which brings further advantages (for instance, coherence and consistency in statements and claims). And the idea that linguistic hypotheses have to be quantitatively assessed whenever it seems possible is becoming more and more common.

\section{Conclusion}

Our analysis of previous studies on how complex sentences with homogeneously collateral sub clauses work in all the functional styles, has given us grounds to infer that our colleagues are mostly interested in the problem of inclusion of connectives between clauses and how they are arranged. The persuasion factor of author's intentionality was largely neglected. On the other hand, it should be noted here that the state-of-the-art in syntactic studies is what pre-defines the ever increasing interest of linguists towards syntactic semantics, in particular, towards the 'language - mind' problem. Referential correlation also provides strong motivation for producing unified models of complex multicomponent syntaxemes with the pronounced 'I' of the author. These properties can be hardly accorded with 'abstract knowledge' about syntactic structures, and it is assumed that (to the highest degree) complex sentences with homogeneously collateral sub clauses are through which "the language of understanding and reproduction" 
is realized but only as one single aspect of the unitary mastery of verbal and cogitative activity of an individual [29].

This paper deals with only some of the questions pertaining to the study and description of the semantic and syntactic characteristics of complex sentences with homogeneously collateral sub clauses, in terms of the persuasive function of the 'I' of the author. However, in this study we established that such structures, combining in themselves expressiveness, emotionality and evaluativity and functioning in practically each and every style, 'push' the addressee to perceive the world in a figurative way because they allow authors to construct individually marked syntaxemes, evaluate the extralinguistic situation of communication, enter internal communication, enhance expressivity - the one that is the driver of language development.

\section{References}

1. I.A. Sternin Practical rhetoric: A study guide for university students. Basic definitions of the science of speech persuasion. Methods of persuasion. 272. (2008)

2. E.V. Sheleshtyuk Speech persuasion: research ontology and methodology. A monograph 344. (2014)

3. I.A. Sternin Practical rhetoric: guidance and exercises for those who strive to speak properly. 155 (2005)

4. T.G. Khazagerov, L.S. Shirina General Rhetoric. 278 (1987)

5. E. Shapovalova, T. Mikheeva Communicative and functional aspects of Russian idioms Academic Publishing 134. (2014)

6. Brent Strickland, Matthew Fisher, Frank Keil, Joshua Knobe Syntax and intentionality: An automatic link between language and theory-of-mind, Cognition, 133, 1, 249-261. Retrieved from: http//www.sciencedirect.com/science/article/pii/S001 0027714001103 (2014)

7. N.S. Piskunov Differential and integral calculus: $A$ handbook, I. 123-124 (1996)

8. A.A. Logunov M.A. Mestvirishvili, O.A. Khrustalev, Constraints on the behaviour of elastic and nonelastic cross-sections at high energy, 153-189 (1971)

9. B.N. Morenko, L.D. Babakova, O.M. Voskerchyan, Urgent issues and problems of studying Russian as a foreign language and teaching in Russian How the Russian-language image of the world is developed in foreign students, , 3-7 (2016)

10. Yu.N. Varzonin A rhetorical model: worldview, ethics, communication Language, culture and society in the humanitarian paradigm: A collection of papers 7-12 (1999)

11. L.G. Ramazanova Metaphors in journalism: a case study of A.N.Tolstoy writings. Author's summary, PhD Thesis in philology. 24. (2004)
12. G.N. Petrakovich A collection of papers, Bio-field uncovered. 305 (2009)

13. O.E. Zakharchuk A monograph-Don, Functional and semantic characteristics of the conceptual and terminological field of stylistics, 141. (2013)

14. O.M. Ayvankhov Izvor, Faith that moves mountains. Selected writings, 238, 34-37 (2006)

15. O.E. Zakharchuk Ecclesiastical-religious style: functional and semantic aspects of study in the system of stylistic coordinates. Language. Discourse. Text, 194-196 (2009)

16. V.I. Karasik Evaluative motivation, the status of an individual and verbal personality, 27-28 (1994)

17. N.Yu. Shvedova Essays on the syntax of the Russian spoken language: A handbook, 378 (1960)

18. I.N. Cheplygina Linguistic means of expressiveness in V.Nabokov's prose. Author's abstract, PhD Thesis in Philology, 21 (2002)

19.E.I. Dibrova, The language system and its development in time and space, na materiale rasskazov A.P. Chekhova, Semantic fields and author's projections in fiction (exemplified by Anton Chekhov's novels). 313-321 (2001)

20. V. Zusman Literary Issues, Concept in the system of humanitarian studies, 2, 3-29 (2003)

21. A.A. Kremnev, «Bulat». Horizons in the green prairies: Poems, 106 (2011)

22. O.V. Nikolenko Semantic variation in complex sentences with homogeneously collateral sub-clauses in the Russian language: PhD Thesis in Philology, 158 (2007)

23. O.V. Nikolenko Cross-disciplinary aspects in linguistic studies Expressiveness in complex sentences with homogeneously collateral sub-clauses 245-253 (2009)

24. M.Yu. Lermontov Selected works, 117-118 (1981)

25. V.A. Shitov Russian syntax issues An attempt of classification of sub clauses by the number of subordinating conjunctions or connectives in them, 76-86. (1969)

26. G.F. Gavrilova Expanded complex sentence in the Russian language 232. (1979)

27. G.F. Kalashnikova Syntax of the multi-component complex sentence in the system of philological education at secondary and tertiary school. A study guide in four parts 296 (1998)

28. G.F. Gavrilova Humanities: objects, regularities, scientific paradigms: A collection of papers, Transitivity in the system of complex sentences with homogeneously collateral subclauses. 3-11 (1998)

29. N. Chater, M. Stewart McCauley, H. Morten Christiansen Language as skill: Intertwining comprehension and production, Journal of Memory and Language, 89, August 2016, 244-254. Retrieved from:http://www.sciencedirect.com/science/article/pi i/S0749596X 